\title{
Cloud Computing Adoption at Higher Education Institutions in the Republic of Sudan
}

\author{
Hussien Abdulatif $^{1^{*}}$, Mosaab Nouraldein Mohammed Hamad ${ }^{2}$ \\ ${ }^{1}$ Faculty of Engineering, Elsheikh Abdallah Elbadri University, Berber, Sudan \\ ${ }^{2}$ Faculty of Health Science, Medical Laboratory Department, Elsheikh Abdallah Elbadri University, Berber, Sudan
}

DOI: $10.36348 /$ sjet.2020.v05i07.002

| Received: 01.07.2020 | Accepted: 10.07.2020 | Published: 14.07.2020

*Corresponding author: Hussien Abdulatif

Abstract

Education today is becoming completely associated with the Information and Communication Technology (ICT) on the content delivery, communication and collaboration. Cloud computing simply involves the provision of information technology (IT) solutions as a service rather than as a product through the Internet. Sudan just like any other developing countries is yet to fully adopt cloud based systems according to trust and security challenges. The motive of this paper is to identify the extent and characteristics of Cloud Computing adoption in Sudanese universities and higher education institutions. The study assessed Cloud Computing uses in terms of actual use of cloud services and applications, ability of transition from conventional systems to cloud computing technology, and efficiency of the technical staff of IT to deal with modern technologies. The adoption of the Cloud Computing services in higher education in Sudan was relatively low. The results showed that here is a significant relationship between the adoption of Cloud Computing in Sudan and the four independent variables; (Basic knowledge of cloud computing services and applications, the actual use of cloud computing technology, ability of transition from conventional systems to Cloud Computing Technology and skills of IT human resources) at level of significance $\alpha=0.05$.

Keywords: Cloud Computing, Higher education, Sudanese Universities.

Copyright @ 2020: This is an open-access article distributed under the terms of the Creative Commons Attribution license which permits unrestricted use, distribution, and reproduction in any medium for non-commercial use (NonCommercial, or CC-BY-NC) provided the original author and source are credited.

\section{BACKGROUND}

Higher education was acknowledged in time as one of the pillars of society development. Through the partnerships between universities, government and industry, researchers and students have proven their contribution to the transformation of society and the entire world economy [1]. Educational instructions all through the World have turned out to be exceptionally needy on information innovation to benefit their business prerequisites. Getting and keeping up an extensive variety of hardware and software require generous, ongoing venture and the aptitudes to help them [2]. Over the previous decade, education industries have developed essentially in the Sudan. This improvement in education and confronted challenges, for example, absence of government spending on education and the loss of educators authorities on account of movements and there is deficiency of qualified instructors in the nation [3].

\section{CLOUD DEFINITION}

Here we have considered the standard definition which was proposed by the U.S. National
Institute of Standards and Technology (NIST): "Cloud computing is a model for enabling ubiquitous, convenient, on-demand network access to a shared pool of configurable computing resources (e.g., networks, servers, storage, applications, and services) that can be rapidly provisioned and released with minimal management effort or service provider interaction" [4].

The accompanying five attributes, as characterized by NIST, are viewed as characteristic in cloud computing services [5]:

1. On-Demand Self-Service: Clients can naturally arrangement computing capacities and assets all alone when required without requiring any human intercession.

2. Broad Network Access: Access and capacities are accessible over the system through standard gadgets, for example, phones, tablets, and so forth.

3. Resource Pooling: Resources, for example, organize data transmission, virtual machines, memory, handling power, storage limitation, and so on are pooled together to serve various clients utilizing a multi-occupant display. That is; virtual 
and physical resources are powerfully allotted and reassigned in light of need and clients' requests.

4. Rapid Elasticity: Contingent upon request, resources and abilities can be rapidly and consequently sent and scaled at any amount and whenever.

5. Measured Service: Client use of the seller's resources and services are consequently checked; controlled and detailed offering an abnormal state of straightforwardness for the client and merchant.

\section{Cloud Service Models}

In cloud wording, the expression "as-aservice" is widely utilized, which basically implies that a given cloud item is offered in a way that it can be "leased" by buyers over the Internet. It is frequently depicted as an "on request" service since it is accessible at whatever point you require it [6].

In reference to NIST definition of cloud computing, there are three typical kinds of cloud computing services:

Software as a Service (SaaS): In this model end-users are provided access to application and software over the internet as a service. The user does not need to install or update software or upgrade hardware in its own device [7].

1. Platform as a Service (PaaS): These models provide the environment of developing and executing application. PaaS contains tools for developing, testing, deploying, and maintaining applications [7].

2. Infrastructure as a Service (IaaS): With an IaaS display, the service supplier conveys the fundamental hardware resources (network, compute, storage) required to run a client's applications. IaaS service models enable clients to exploit these hugely adaptable networks and server farms at a small amount of the cost related with building and dealing with their own particular foundations [8].
Fig-1 illustrates the cloud service models.

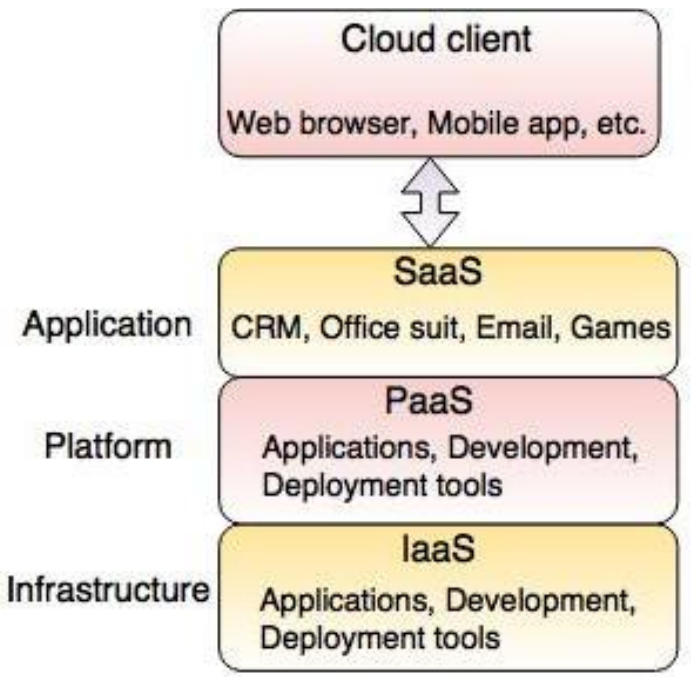

Fig-1: Cloud service models [9]

\section{Cloud Deployment Models}

This refers to the location and management of the cloud's infrastructure. The cloud environment is subdivided into public, private, hybrid and community clouds:

1. Public Cloud: is this in which services are available to the general public or a large industry group and is owned by an organization selling cloud services. In the public cloud services are available at large over the Internet [10].

2. Private Cloud: The cloud infrastructure is hosted and managed by the organization or a third party [11].

3. Community Cloud: The cloud infrastructure is shared by a number of organizations have similar requirements [11].

4. Hybrid Cloud: The cloud infrastructure is a combined of two or more clouds like private, community, or public [11].

Fig-2 illustrates the models of cloud deployment.

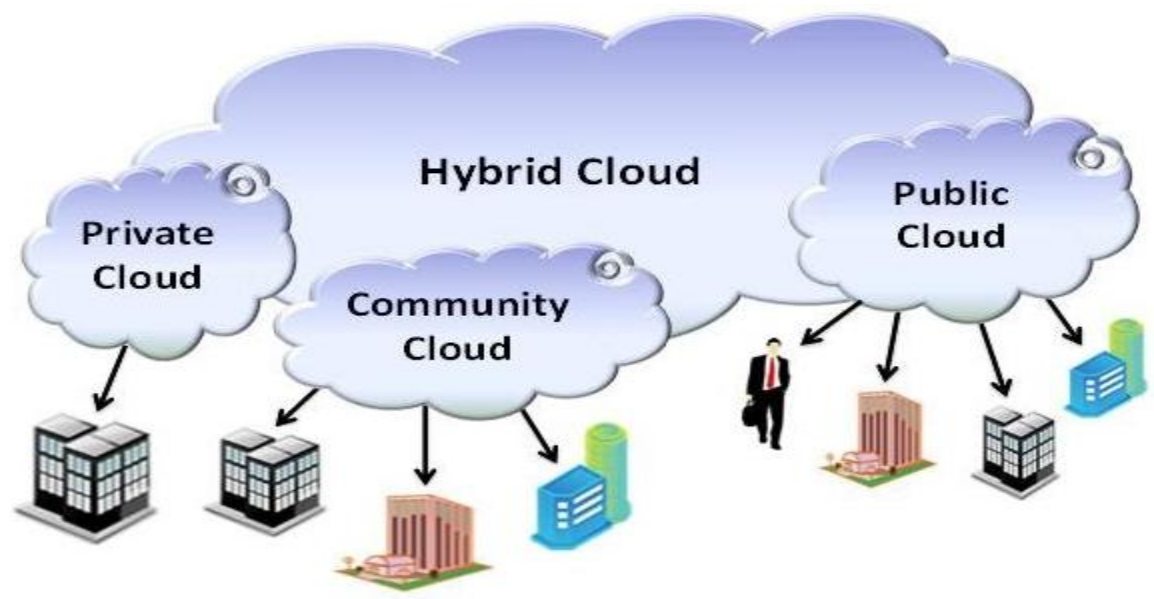

Fig-2: Cloud deployment models [12] 
Hussien Abdulatif \& Mosaab Nouraldein., Saudi J Eng Technol, July., 2020; 5(7): 295-299

\section{LITERATURE REVIEW}

There are some of related works that have been done in different countries around the worlds. Askari et $a l$. , in [13] proposed a cloud computing building design for higher education association containing the different deployment models, service models and client area. Finally authors provided suggestions to a productive and effective relocation from standard to cloud based framework. In Nayar et al., [14] analyzed the approach that has been followed to identify the benefits and restrictions of cloud computing. Specific case of a lab setup has been taken to illustrate the money related aspects. A comparative investigation of cloud-based facilitating versus conventional (on-premise) application deployment has additionally been presented. Senyo et al., in [15] indicated that extant cloud computing literature tends to skew towards the technological dimension to the detriment of other under researched dimensions, for example, business, conceptualization and application space. While there has been a consistent increase in cloud computing studies over the most recent seven years, a noteworthy number of these studies have not been underpinned by theoretical frameworks and models. In Al-Shqeerat et al., [16] presented the advantages of cloud computing technology in education sector, and explained the security challenges that meet educational institution when adopting cloud technology. The authors provide some basic recommendations to overcome these challenges such as a strong encryption, backup and recovery schemes that must be used to save data and proper security mechanisms should be used to prevent the access to this data.

After this brief literature review providing the context from the infrastructure, application and services aspect of cloud computing, this paper focuses on reflecting the current situation of using cloud computing services and applications in higher educational institutions in Sudan and ability to transition from traditional system to cloud technology.

\section{Data Collection}

In the wake of assessing the writing and meeting the experts, the questionnaire is the most suitable device to meet the objective of this research. The research population consists of the managers of IT staff in each university. An online questionnaire is distributed to group of IT staff in different higher education institutions in Sudan; which represents the research population, (19) are retrieved.

\section{Data Analysis}

To achieve the research goal, the researcher would use data analysis both qualitative and quantitative data analysis methods. The Data analysis will be made utilizing (SPSS 20). The researcher would utilize the following statistical tools:

1. Pearson correlation coefficient for Validity.

2. Cronbach's Alpha for Reliability Statistics.

3. Frequency and Descriptive analysis.

4. Parametric Tests (One-sample T test).

\section{Structure Validity of the Questionnaire}

Structure validity is the second statistical test that is used to test the validity of the questionnaire structure by testing the validity of each field and the validity of the whole questionnaire. It measures the correlation coefficient between one field and all the fields of the questionnaire that have the same level of likert scale.

\section{RESULTS}

Table-1 clarifies the correlation coefficient for each field and the whole questionnaire. The $\mathrm{p}$-values (Sig.) are less than 0.05 , so the correlation coefficients of all the fields are significant at $\alpha=0.05$, so it can be said that the fields are valid to measure what it was set for to achieve the main aim of the research.

Table-1: Correlation coefficient of each field and the whole of questionnaire

\begin{tabular}{|l|l|l|l|}
\hline No & Paragraph & $\begin{array}{l}\text { Pearson Correlation } \\
\text { coefficient }\end{array}$ & $\begin{array}{l}\text { P-Value } \\
(\text { sig })\end{array}$ \\
\hline 1 & Basic knowledge of cloud computing services and applications & $\mathbf{. 8 1 3}$ & $\mathbf{. 0 0 0}$ \\
\hline 2 & The actual use of cloud computing technology & $\mathbf{. 8 8 8}$ & $\mathbf{. 0 0 0}$ \\
\hline 3 & $\begin{array}{l}\text { Ability of transition from conventional systems to Cloud Computing } \\
\text { Technology }\end{array}$ & $\mathbf{. 7 6 7}$ & $\mathbf{. 0 0 0}$ \\
\hline 4 & Skills of IT human resources. & $\mathbf{. 7 3 9}$ & $\mathbf{. 0 0 0}$ \\
\hline
\end{tabular}

*Correlation is significant at $\mathbf{0 . 0 5}$ level.

\section{Reliability of the questionnaire}

This method is used to measure the reliability of the questionnaire between each field and the mean of the whole fields of the questionnaire. The normal range of Cronbach's coefficient alpha value between 0.0 and +1.0 , and the higher values reflects a higher degree of internal consistency. The Cronbach's coefficient alpha was calculated for each field of the questionnaire.
Table-2 shows the values of Cronbach's Alpha for each field of the questionnaire and the entire questionnaire. For the fields, values of Cronbach's Alpha were in the range from 0.702 and 0.909 . This range is considered high; which indicates an excellent reliability of each field of the questionnaire. 
Table-2: Cronbach's Alpha for each field of the questionnaire

\begin{tabular}{|l|l|l|}
\hline No & paragraph & Cronbach's Alpha \\
\hline $\mathbf{1}$ & Basic knowledge of cloud computing services and applications & $\mathbf{. 8 1 1}$ \\
\hline $\mathbf{2}$ & The actual use of cloud computing technology. & $\mathbf{. 9 0 9}$ \\
\hline $\mathbf{3}$ & Ability of transition from conventional systems to Cloud Computing Technology & $\mathbf{. 9 0 9}$ \\
\hline $\mathbf{4}$ & Skills of IT human resources. & $\mathbf{. 7 0 2}$ \\
\hline
\end{tabular}

\section{DISCUSSION}

Through the results of the statistical analysis of the respondent's views, the most important findings of this study could be summarizing as following:

- With regard to "Basic knowledge of cloud computing services and applications"

According to statistical analysis, the research is reached to the following conclusions:

1. There is $81 \%$ of the respondents has good knowledge of the services provided by cloud computing. In addition $80 \%$ of respondents have good knowledge of cloud computing applications provided by companies, such as Google drive and others.

2. There is $79 \%$ of the respondents has good knowledge of the virtualization. And $73.6 \%$ of respondents have has knowledge of Cloud Computing Technology.

3. In general, there is $78.4 \%$ of respondents has good knowledge about cloud computing technology which is the one of good points to adopt this new technology.

- With regard to "The actual use of Cloud Computing Technology"

According to statistical analysis, the research is reached to the followings:

1. There is $68.4 \%$ of the respondents see that the services of cloud computing are a useful. On other hand, there is $46.4 \%$ of respondents see that institution gets a good benefit from cloud computing technology. Also, $43.2 \%$ of respondents see that their university adopts electronic cloud depending on technical assistance.

2. There is $40 \%$ of the respondents see that there is a good adoption of cloud computing services in their institution. In addition, $37.8 \%$ of respondents see that the quality of services provided is good and $35.8 \%$ of the respondents see that the existing cloud computing in their institution has good services.

3. In general, there is $45.27 \%$ of respondents see that there is real using of cloud computing technology in their institution, which means that the existence of cloud technology in educational institutions is very weak.
- With regard to "Ability of transition from conventional systems to Cloud Computing Technology"

According to statistical analysis, the research is reached to the following conclusions:

1. There is $80 \%$ of the respondents see that the institution has high speed internet lines, which is the one of the concerns to adopt cloud computing. In addition there are $72.6 \%$ of the respondents assure that there good quality services provided by telecommunications companies in this institution.

2. There is $69.4 \%$ of the respondents see that current services support the existing technology in institution. Moreover, $68.4 \%$ of respondents see that the existing computer network has global standards.

3. There is $67.4 \%$ of the respondents see that of the current network has ability to add accounts for the wide range, and existing infrastructure provide good services.

4. There is $66.4 \%$ of the respondents agree that existing infrastructure able to transition to cloud computing.

5. There is $65.2 \%$ of the respondents see that current electronic system able to storage all data. On the other hand, there is $60 \%$ of the respondents see that the system has good level of confidentiality and data-keeping.

6. In general, there is $68.5 \%$ of respondents support the idea of transition from conventional systems to Cloud Computing Technology.

- With regard to " Skills of IT human resources"

According to statistical analysis, the research is reached to the followings:

1. There is $85.2 \%$ of the respondents agree that transition to cloud computing technology will increase the skills and efficiency of the IT staff. In addition $74.8 \%$ of the respondents see that current efficiency of the IT staff is sufficient to deal with all that is new in this field.

2. As voiced, $52.6 \%$ of respondents see that the institution provide training opportunities to IT staff on modern systems. 
In general, medium approval of $70.87 \%$ of respondents see that skills of IT staff is sufficient to deal with adopting of cloud computing technology.

\section{CONCLUSION}

Higher Education institutions play an important role in the growth of societies. As with organizations nowadays, universities have become more reliant on Information and Communication Technology (ICT). ICT and internet based services have to provide their stakeholders with educational services. Cloud computing is likely to be an attractive proposition to start up and small to medium educational establishments. The potential of cloud computing may include but is not limited to increasing service efficiency and cost-savings. The goal of this study is to describe the current usage of cloud computing services in Sudanese Higher education Institutes. The experimental result of this paper presents that adoption of cloud computing services and applications is very weak in most of these higher institutes. In this paper some significant factors has been derived from data collection and data analysis on different institutes that support transition to cloud computing. Lack of resource requirements and proper infrastructure are found important. Other important factor is lack of training opportunities to IT staff on modern systems.

\section{RECOMMENDATIONS}

The findings obtained from this research would assist in providing a roadmap to improve Cloud Computing adoption in higher education institutions in Sudan and the output factors may increase ability of transition from traditional systems to cloud computing technology.

\section{REFERENCES}

1. Mircea, M., \& Andreescu, A. L. (2011). Using Cloud Computing in Higher Education: A Strategy to Improve Agility in the Current Financial Crisis. Communications of the IBIMA, 15.

2. Sclater, N. (2010). Cloud computing in education, Policy Brief, Unesco Institute for Information Technology in Education.

3. Adrees, M. S., Omer, M. K. A., \& Sheta, O. E. (2015). Cloud computing architecture for higher education in the third world countries (republic of the Sudan as model). International Journal of Database Management Systems, 7(3):13-24.

4. Peter, M., \& Timothy, G. (2011). The NIST Definition of Cloud Computing. National Institute of Standards and Technology Special Publication. 800-145.

5. Seke, M. M. (2015). Higher education and the adoption of cloud computing technology in Africa.
International Journal on Communications, 1(9):19.

6. Landis, C., \& Blacharski, D. (2013). Cloud Computing Made Easy. Vitual Global, Incorporated.

7. Osman, A., Abdulatif, H., El-Gelany, A., \& Alias, N. (2017). Private Cloud: Effective Strategy in Developed Countries Case Study: Sudanese Universities. IOSR Journal of Computer Engineering (IOSR-JCE), 19(2):73-79.

8. Mather, T., Kumaraswamy, S., \& Latif, S. (2009). Cloud security and privacy: an enterprise perspective on risks and compliance. "O'Reilly Media, Inc.".

9. https://www.tutorialride.com/cloudcomputing/service-models-in-cloud computing.htm, [Online; access 16-May-2020].

10. Maraschini, A., Levy, E., \& Rochwerger, B. (2013). Towards Cloud Computing at IS Department, King Abdulaziz Univeristy. Middle Eastern \& African Journal of Educational Research, 4, 4-11.

11. Piplode, R., \& Singh, U. K. (2012). An overview and study of security issues \& challenges in cloud computing. International Journal of Advanced Research in Computer Science and Software Engineering, 2(9).

12. Bamiah, M. A. (2015). Trusted Cloud Computing Frame Work in Critical Industrial Application (Doctoral dissertation, Universiti Teknologi Malaysia).

13. Askari, S. H., Ahmad, F., Umair, S., \& Khan, S. A. (2018). Cloud Computing Education Strategies: A Review. In Exploring the Convergence of Big Data and the Internet of Things (pp. 43-54). IGI Global.

14. Nayar, K. B., \& Kumar, V. (2018). Cost benefit analysis of cloud computing in education. International Journal of Business Information Systems, 27(2), 205-221.

15. Senyo, P. K., Addae, E., \& Boateng, R. (2018). Cloud computing research: A review of research themes, frameworks, methods and future research directions. International Journal of Information Management, 38(1), 128-139.

16. Al-Shqeerat, K. H., Al-Shrouf, F. M., Hassan, M. R., \& Fajraoui, H. (2017). Cloud computing security challenges in higher educational institutions-A survey. International Journal of Computer Applications, 161(6), 22-29.

17. Hashim, Y. E., \& Talab, E. A. (2017). State-ofthe-art cloud computing in Sudanese higher education institutions. International Advanced Research Journal in Science, Engineering and Technology. 4(9):236-242. 